\title{
Stability of Matter in Magnetic Fields
}

\author{
Elliott H. Lieb ${ }^{1,2}$, Michael Loss ${ }^{3}$ and Jan Philip Solovej ${ }^{2}$ \\ ${ }^{1}$ Department of Physics, Jadwin Hall, Princeton University, P. O. Box 708, Princeton, New Jersey 08544 \\ ${ }^{2}$ Department of Mathematics, Fine Hall, Princeton University, Princeton, New Jersey 08544 \\ ${ }^{3}$ School of Mathematics, Georgia Institute of Technology, Atlanta, Georgia 30332
}

April 11 (revised June 11)

\begin{abstract}
In the presence of arbitrarily large magnetic fields, matter composed of electrons and nuclei was known to be unstable if $\alpha$ or $Z$ is too large. Here we prove that matter is stable if $\alpha<0.06$ and $Z \alpha^{2}<0.04$.
\end{abstract}

One of the remaining unsolved problems connected with the stability of matter is the inclusion of arbitrary magnetic fields. The model is a caricature of QED which invites speculations about stability of QED for large fine structure constant, $\alpha$, but that is not our focus here and we refer to [1] for a discussion of these and related matters. The Hamiltonian for $N$ electrons and $K$ fixed nuclei of charge $Z e$ with magnetic field $\boldsymbol{B}(x)=\nabla \times \boldsymbol{A}(x)$, including the field energy, $\varepsilon \int B^{2}$, is

$$
H=\sum_{i=1}^{N} \mathcal{T}_{i}+V_{\mathrm{c}}+\varepsilon \int B(x)^{2} \mathrm{~d}^{3} x
$$

where $\mathcal{T} \equiv[\boldsymbol{\sigma} \cdot(\boldsymbol{p}+\boldsymbol{A})]^{2}=(\boldsymbol{p}+\boldsymbol{A})^{2}+\boldsymbol{\sigma} \cdot \boldsymbol{B}$ is the Pauli operator. The Coulomb energy is

$$
\begin{aligned}
V_{\mathrm{c}}= & -Z \sum_{i=1}^{N} \sum_{j=1}^{K}\left|x_{i}-R_{j}\right|^{-1}+\sum_{1 \leq i<j \leq N}\left|x_{i}-x_{j}\right|^{-1} \\
& +Z^{2} \sum_{1 \leq i<j \leq K}\left|R_{i}-R_{j}\right|^{-1}
\end{aligned}
$$


with $R_{j}$ being the coordinates of the nuclei and $x_{i}$ the electron coordinates. The energy unit is 4 Rydbergs $=2 m c^{2} \alpha^{2}, \alpha=e^{2} / \hbar c$, length unit $=\hbar^{2} / 2 m e^{2}$ and $\varepsilon=\left(8 \pi \alpha^{2}\right)^{-1}$. Notice that $\alpha$ appears in (11) only through $\varepsilon$.

The negative particles, i.e., the electrons, are necessarily spin 1/2 fermions which, for generality, we assume to exist in $q / 2$ flavors (e.g., $q=6$ ). The ground state energy is denoted by $E$.

Starting with the 1967 pioneering work of Dyson and Lenard we now understand stability for arbitrarily many electrons and nuclei, with $\boldsymbol{B}=0$, in the context of the nonrelativistic Schrödinger equation. Later it was extended to the "relativistic" Schrödinger equation in which $\boldsymbol{p}^{2} / 2 m$ is replaced by $\left(c^{2} \boldsymbol{p}^{2}+m^{2} c^{4}\right)^{1 / 2}$ (see [2] for a review). These proofs also hold with the inclusion of a magnetic field coupled to the orbital motion of the electrons, i.e., $\boldsymbol{p} \rightarrow \boldsymbol{p}+\boldsymbol{A}$, but no Zeeman $\boldsymbol{\sigma} \cdot \boldsymbol{B}$ term.

Stability of matter has two meanings: (1) $E$ is finite for arbitrary N and K; (2) $E \geq$ $-C_{1}(N+K)$ for some constant $C_{1}$ independent of $N, K$ and $R_{j}$. In the nonrelativistic case (2) holds. In the relativistic case, (1) implies (2) but (1) requires two conditions: $Z \alpha \leq C_{2}$ and $\alpha \leq C_{3}$ with $C_{2}$ and $C_{3}$ being universal constants, the best available values being in [3], Theorems 1 and 2. The inclusion of $\boldsymbol{B}$ changes $E$, but the point is that while $C_{1}, C_{2}, C_{3}$ depend on $q$, they can be chosen to be independent of $\boldsymbol{B}$.

The situation changes dramatically when the magnetic moments of the electrons are allowed to interact with the magnetic field via the $\boldsymbol{\sigma} \cdot \boldsymbol{B}$ term, as in (1). The reason for this is simple: The Pauli operator $\mathcal{T}$ is nonnegative but it is much weaker than $(\boldsymbol{p}+\boldsymbol{A})^{2}$. Indeed, it can even have square integrable zero-modes [4], $\mathcal{T} \psi=0$, for suitable $\boldsymbol{A}(x)$, which cause instability for large $Z \alpha^{2}$.

It is known [5] that without the field energy term, $\varepsilon \int B^{2}$, in (11) arbitrarily large $\boldsymbol{B}$ fields can cause arbitrarily negative energies, $E$, even for hydrogen. The field energy, hopefully, stabilizes the situation, and our goal is to show that $E$ is finite for (1), even after minimizing over all possible $\boldsymbol{B}$ fields and all possible $R_{j}$.

One of our results on magnetic stability is:

Theorem 1: The ground state energy of $H$ satisfies

$$
E \geq-2.6 q^{2 / 3} \max \left\{Q(Z)^{2}, Q(5.7 q)^{2}\right\} N^{1 / 3} K^{2 / 3}
$$

with $Q(t) \equiv t+\sqrt{2 t}+2.2$, provided that

$$
q Z \alpha^{2} \leq 0.082 \quad \text { and } \quad q \alpha \leq 0.12
$$


In (2) all the nuclear charges are set equal to $Z$. As far as stability is concerned this is no restriction [6] since the energy is concave in each charge $Z_{j}$ and hence stability holds in the "cube" $\left\{0 \leq Z_{j} \leq Z\right\}_{j=1}^{K}$ if it holds when all $Z_{j}=Z$. It also follows from this that $E$ is a decreasing function of $Z$. Moreover, since $\varepsilon \propto \alpha^{-2}$ it follows that $E$ is a decreasing function of $\alpha$, a fact that will be important later. The form of (3) is the best possible for $Z \geq 1$, as we know from other studies [2].

Our actual condition for stability given after (18) is rather complicated, but very much more general than (4) - which is only representative. The results after (18) show, e.g., that when $\alpha=1 / 137$, and $q=2, Z$ can be as large as 1050. The large values of $Z$ and $\alpha$ are important because the comfortable distance of the critical values from the physical values $Z \leq 92, \alpha=1 / 137$ implies that the effect studied here is merely a small perturbation.

Our proof of Theorem 1 will require a new technique - a running energy-scale renormalization of $\mathcal{T}$. A by-product of this is a Lieb-Thirring type inequality for $\mathcal{T}$ :

Theorem 2: If $\varepsilon_{1} \leq \varepsilon_{2}, \ldots<0$ are the negative eigenvalues of $\mathcal{T}-U$, for a potential $-U(x) \leq 0$ then

$$
\begin{aligned}
\sum\left|\varepsilon_{i}\right| & \leq a_{\gamma} \int U(x)^{5 / 2} \mathrm{~d}^{3} x \\
& +b_{\gamma}\left(\int B(x)^{2} \mathrm{~d}^{3} x\right)^{3 / 4}\left(\int U(x)^{4} \mathrm{~d}^{3} x\right)^{1 / 4}
\end{aligned}
$$

for all $0<\gamma<1$, where $a_{\gamma}=\left(2^{3 / 2} / 5\right)(1-\gamma)^{-1} L_{3}$ and $b_{\gamma}=3^{1 / 4} 2^{-9 / 4} \pi \gamma^{-3 / 8}(1-\gamma)^{-5 / 8} L_{3}$. We can take $L_{3}$, defined below, to be 0.1156 .

More generally the second term in (5) can be replaced by $\left(\int B^{3 q / 2}\right)^{1 / q}\left(\int U^{p}\right)^{1 / p}$, where $p^{-1}+q^{-1}=1$.

The investigation of this problem started in [1, 7] where type 2 stability was proved (for suitable $Z, \alpha$ and $q=2$ ) for $K=1$ and arbitrary $N$ (if $(Z+1 / 4) \alpha^{12 / 7} \leq 0.15$ ) or $N=1$ and arbitrary $K$ (if $Z \alpha^{2} \leq 0.6$ and $\alpha \leq 0.3$ ). The problem for general $N$ and $K$ was open for 9 years and we present a surprisingly simple solution here.

The bounds in (4) on $Z \alpha^{2}$ and $\alpha$ are not artifacts. It is shown in [1] and [国] that the zero-modes cause $E=-\infty$ when $Z \alpha^{2}>11.11$ for the "hydrogenic" atom, i.e., a single spin $1 / 2$ particle and one nucleus. If the number of nuclei is arbitrary, it is shown in [7] that there is collapse if $\alpha>6.67$, no matter how small $Z$ is. Magnetic stability, like relativistic stability, implies a (Z-independent) bound on $\alpha$.

Prior to our work a proof of type 2 stability for (1) with $Z=1, q=2$, and some sufficiently small $\alpha$ was announced (unpublished) by C. Fefferman and sketched to one of us. 
Our proof is unrelated to his, considerably simpler and, more importantly, gives physically realistic constants.

We begin our analysis with the observation that length scaling considerations suggest that the key to understanding the stability problem is somehow to replace $\mathcal{T}$, on each energy scale, $e$, by $\mu \mathcal{T} / e$, where $\mu$ is a fixed energy but $e$ is variable. On energy scales $e>\mu$ we can use the fact that $\mathcal{T}>0$ to replace $\mathcal{T}$ by $\mu \mathcal{T} / e$ without spoiling lower bounds. It might seem odd to replace $\mathcal{T}$ by something smaller, but what is really happening is that $\boldsymbol{\sigma} \cdot \boldsymbol{B}$ is being partially controlled by $\left[1-\mu e^{-1}\right](\boldsymbol{p}+\boldsymbol{A})^{2}$. The idea of replacing $\mathcal{T}$ by a fraction of $\mathcal{T}$ was also used in [1], but no energy dependence was used there.

We shall illustrate this concept by three calculations. The first, A, will establish magnetic stability by relating it to the stability of relativistic matter (see [3, 6, 8, 9]). The second, B, will be the proof of Theorem 2. The third, C, will use essential parts of the second calculation and an electrostatic inequality proved in [3] to prove magnetic stability without resorting to relativistic stability.

A. Magnetic Stability from Relativistic Stability: We use stability of relativistic matter in the form proved in [3]. From the corollary of Theorem 1 in [3] with $\beta=0.5$ we have, for any $0<q \kappa \leq 0.032$ and $Z \kappa \leq 1 / \pi$,

$$
\sum_{i=1}^{N}\left|\boldsymbol{p}_{i}+\boldsymbol{A}_{i}\right|+\kappa V_{\mathrm{c}} \geq 0 .
$$

(Although Theorem 1 in [3] was stated only for $|\boldsymbol{p}|$, it holds for $|\boldsymbol{p}+\boldsymbol{A}|$ because it relies only on the magnitude of the resolvent which only gets smaller when $\boldsymbol{A}$ is not zero. That is , || $\boldsymbol{p}+\left.\boldsymbol{A}\right|^{-s}(x, y)|\leq||\boldsymbol{p}|^{-s}(x, y) \mid$ for each $s>0$ and $x, y$ in $\boldsymbol{R}^{3}$. This follows at once from a similar bound on the heat kernel $\left\{\exp \left[-t(\boldsymbol{p}+\boldsymbol{A})^{2}\right]\right\}(x, y)$ which, in turn, follows from its representation as a path integral. This was pointed out in [5, 10]. Only the resolvent powers $|\boldsymbol{p}+\boldsymbol{A}|^{-s}$ enter the proof of Theorem 1 in [3].)

Using (6), $H$ is bounded below by $\bar{H}=\sum_{i=1}^{N} h_{i}$ where $h$ is the one-body operator $h=$ $\mathcal{T}-\kappa^{-1}|\boldsymbol{p}+\boldsymbol{A}|$. Thus, $E$ is bounded below by $\varepsilon \int B^{2}+\bar{E}_{N}$, where $\bar{E}_{N}=q \sum_{j=1}^{[N / q]} \varepsilon_{j}$ and $\varepsilon_{1} \leq \varepsilon_{2} \leq \ldots$ are the eigenvalues of $h$. For $e>0$, let $N_{-e}(h)$ be the number of eigenvalues of $h$ less than or equal to $-e$. Choose $\mu>0$ and note that

$$
\bar{E}_{N} \geq-N \mu-q \int_{\mu}^{\infty} N_{-e}(h) \mathrm{d} e .
$$

The crucial step in our proof is noting that the positivity of the operator $\mathcal{T}$ implies that $\mathcal{T} \geq \mu \mathcal{T} / e$ when $e \geq \mu$. Thus, $\mathcal{T} \geq \mu e^{-1} \mathcal{T} \geq \mu e^{-1}(\boldsymbol{p}+\boldsymbol{A})^{2}-\mu e^{-1} B(x)$ when $e \geq \mu$. 
By Schwarz's inequality, $\kappa^{-1}|\boldsymbol{p}+\boldsymbol{A}| \leq(1 / 3) e^{-1} \kappa^{-2}(\boldsymbol{p}+\boldsymbol{A})^{2}+(3 e / 4)$ and hence if we set $\mu=(4 / 3) \kappa^{-2}$ we obtain

$$
h \geq e^{-1} \kappa^{-2}(\boldsymbol{p}+\boldsymbol{A})^{2}-(4 / 3) e^{-1} \kappa^{-2} B(x)-(3 e / 4) \equiv h_{e} .
$$

Thus, $N_{-e}(h) \leq N_{-e}\left(h_{e}\right)$ and this can be estimated by the Cwikel-Lieb-Rozenblum (CLR) bound [11], i.e., $N_{-e}\left((\boldsymbol{p}+\boldsymbol{A})^{2}-U(x)\right) \leq L_{3} \int[U(x)-e]_{+}^{3 / 2} \mathrm{~d}^{3} x$ where $[a]_{+} \equiv \max (a, 0)$ and $L_{3}=0.1156$. In our case:

$$
N_{-e}\left(h_{e}\right) \leq L_{3} \int\left[\frac{4 B(x)}{3}-\frac{e^{2} \kappa^{2}}{4}\right]_{+}^{3 / 2} \mathrm{~d}^{3} x .
$$

Inserting this bound in (7), a simple calculation yields

$$
\bar{E}_{N} \geq-N \mu-(2 \pi / 3) q \kappa^{-1} L_{3} \int B(x)^{2} \mathrm{~d}^{3} x .
$$

We choose $\kappa$ so that the field energy terms are non-negative, i.e., $\kappa \geq\left(16 \pi^{2} / 3\right) L_{3} \alpha^{2} q=$ $6.1 \alpha^{2} q$. We conclude, by (6), that magnetic stability holds if

$$
q \alpha \leq 0.071 \text { and } q Z \alpha^{2} \leq 0.052
$$

For $q=2$, the first condition is $\alpha \leq 1 / 28$. For $q=2$ and $\alpha=1 / 137$ stability occurs if $Z \leq 490$.

Assuming (9) holds, we then use (6) and choose $\kappa=\min \left\{0.0315 q^{-1},(\pi Z)^{-1}\right\}$. Our lower bound on the ground state energy per electron, by this method, is then $-\mu=-(4 / 3) \kappa^{-2}=$ $-\max \left\{1345 q^{2}, 13.2 Z^{2}\right\}$.

Remark: We used the CLR bound in (8). Since the derivation of this bound is not elementary the reader might wish to use an easier to derive bound - at the cost of worsening the final constants. A useful substitute is

$$
N_{-e} \leq 0.1054 e^{-1 / 4} \int[U(x)-e / 2]_{+}^{7 / 4} \mathrm{~d}^{3} x
$$

which is in (2.8) of [12] and which can be derived by means originally employed for the Lieb-Thirring inequality. This same remark also applies to our other calculations below.

B. The Lieb-Thirring Inequality: As before we note that $\sum \varepsilon_{i}=-\int_{0}^{\infty} N_{-e}(\mathcal{T}-U) \mathrm{d} e$. We write $\int_{0}^{\infty}=\int_{0}^{\mu}+\int_{\mu}^{\infty}$. The parameter $\mu$ will be optimized below. Noting that $\mathcal{T} \geq$ $(\boldsymbol{p}+\boldsymbol{A})^{2}-B(x)$ and applying the CLR bound in the same fashion as before to $\int_{0}^{\mu}$ yields

$$
L_{3} \int_{0}^{\mu} \int[B(x)+U(x)-e]_{+}^{3 / 2} \mathrm{~d}^{3} x \mathrm{~d} e .
$$


In $\int_{\mu}^{\infty}$ we replace $\mathcal{T}$ by the lower bound $\mu e^{-1}\left[(\boldsymbol{p}+\boldsymbol{A})^{2}-B(x)\right]$ and obtain $N_{-e}(\mathcal{T}-U) \leq$ $N_{-e}\left(\mu e^{-1}\left[(\boldsymbol{p}+\boldsymbol{A})^{2}-B\right]-U\right)$. A further application of the CLR inequality yields the bound on $\int_{\mu}^{\infty}$

$$
L_{3} \int_{\mu}^{\infty} \int\left[B(x)+(e / \mu) U(x)-e^{2} / \mu\right]_{+}^{3 / 2} \mathrm{~d} x \mathrm{~d} e .
$$

It is easy to see that for any $0<\gamma<1$ the integrand in (10) is bounded above by

$$
\sqrt{2}\left(\left[B(x)-\gamma e^{2} / \mu\right]_{+}^{3 / 2}+[U(x)-(1-\gamma) e]_{+}^{3 / 2}\right) .
$$

Treating the integrand in (11) in a similar fashion and combining the inequalities we find

$$
\begin{aligned}
\sum\left|\varepsilon_{i}\right| & \leq \sqrt{2} L_{3} \int\left\{\int_{0}^{\infty}\left[B(x)-\gamma e^{2} / \mu\right]_{+}^{3 / 2} \mathrm{~d} e\right. \\
& +\int_{0}^{\mu}[U(x)-(1-\gamma) e]_{+}^{3 / 2} \mathrm{~d} e \\
& \left.+\int_{\mu}^{\infty}\left[(e / \mu) U(x)-(1-\gamma) e^{2} / \mu\right]_{+}^{3 / 2} \mathrm{~d} e\right\} \mathrm{d}^{3} x .
\end{aligned}
$$

After extending the last two integrals to $\int_{0}^{\infty}$, a straightforward computation yields

$$
\begin{aligned}
\sum\left|\varepsilon_{i}\right| \leq \quad & \sqrt{2} L_{3} \int\left\{\frac{2}{5(1-\gamma)} U(x)^{5 / 2}+\frac{3 \pi \mu^{1 / 2}}{16 \gamma^{1 / 2}} B(x)^{2}\right. \\
& \left.+\frac{3 \pi}{128} \mu^{-3 / 2}(1-\gamma)^{-5 / 2} U(x)^{4}\right\} \mathrm{d}^{3} x
\end{aligned}
$$

Optimizing over $\mu$ yields (5).

To prove the more general form of (5) replace $\mu e^{-1}$ by $\left(\mu e^{-1}\right)^{s}$, where $s=2 p / 3-5 / 3$.

C. Proof of Theorem 1: We turn now to our third illustration of the concept of running energy scale and prove the stability directly, not relating it to the relativistic problem. By this method we get the correct dependence of the ground state energy on Z and also somewhat better critical constants than in (9).

Following [3] we first replace the Coulomb potential by a single particle potential in (12) below. We break up $\boldsymbol{R}^{3}$ into Voronoi cells defined by the nuclear locations, i.e., $\Gamma_{j}=\{x$ : $\left|x-R_{j}\right| \leq\left|x-R_{k}\right|$ for all $\left.k\right\}$ is the $\mathrm{j}$-th Voronoi cell. Each $\Gamma_{j}$ contains a ball centered at $R_{j}$ with radius $D_{j}=\min \left\{\left|R_{j}-R_{k}\right|: j \neq k\right\} / 2$.

The following bound on $V_{\mathrm{c}}$ is proved in [3]: Choose some $0<\lambda<1$. Then

$$
V_{\mathrm{c}} \geq-\sum_{i=1}^{N} W\left(x_{i}\right)+\sum_{j=1}^{K} \frac{Z^{2}}{8 D_{j}},
$$


where $W(x)=Z\left|x-R_{j}\right|^{-1}+F_{j}(x)$ for $x \in \Gamma_{j}$ with $F_{j}(x)$ defined by

$$
\begin{array}{rll}
\left(2 D_{j}\right)^{-1}\left(1-D_{j}^{-2}\left|x-R_{j}\right|^{2}\right)^{-1} & \text { for } & \left|x-R_{j}\right| \leq \lambda D_{j} \\
(\sqrt{2 Z}+1 / 2)\left|x-R_{j}\right|^{-1} & \text { for } & \left|x-R_{j}\right|>\lambda D_{j} .
\end{array}
$$

The point about this inequality is that the potential $W$ has the same singularity near each nucleus as $V_{c}$ has, and that the rightmost term in (12) is repulsive. This term will be responsible for stabilizing the system.

The problem is thus reduced to obtaining a lower bound on $q \sum^{\prime} \varepsilon_{j}$, where $\sum^{\prime} \varepsilon_{j}$ is the sum of the first $[N / q]$ negative eigenvalues of of $\mathcal{T}-W$. Note that Theorem 2 cannot be applied directly to this problem, since $W$ is neither integrable to the power $5 / 2$ nor to the power 4 . Instead we have to do the calculations directly.

For $\nu>0$ (a number that is chosen later) set $W_{\nu}(x) \equiv(W(x)-\nu)_{+}$and note that $W(x)-\nu \leq W_{\nu}(x)$. Then, as in (17), $q \sum^{\prime} \varepsilon_{j} \geq-N \nu-q \int_{0}^{\infty} N_{-e}\left(\mathcal{T}-W_{\nu}\right) \mathrm{d} e$. Again,

$$
\begin{aligned}
\int_{0}^{\infty} N_{-e}\left(\mathcal{T}-W_{\nu}\right) \mathrm{d} e & \leq \int_{0}^{\mu} N_{-e}\left(\mathcal{T}-W_{\nu}\right) \mathrm{d} e \\
& +\int_{\mu}^{\infty} N_{-e}\left(\mu e^{-1} \mathcal{T}-W\right) \mathrm{d} e
\end{aligned}
$$

where we have replaced $W_{\nu}(x)$ by $W(x)$ in the second term. Applying the CLR bound to the first expression on the right side we obtain $L_{3} \iint_{0}^{\mu}\left[B(x)+W_{\nu}(x)-e\right]_{+}^{3 / 2} \mathrm{ded}^{3} x$ which can be bounded, as in part B, by

$$
\begin{aligned}
\sqrt{2} L_{3} \int\{\quad & \int_{0}^{\mu}\left[B(x)-\frac{\gamma e^{2}}{\mu}\right]_{+}^{3 / 2} \mathrm{~d} e \\
& \left.+\frac{2}{5}(1-\gamma)^{-1} W_{\nu}(x)^{5 / 2}\right\} \mathrm{d}^{3} x
\end{aligned}
$$

for any $0<\gamma<1$.

The difficulty in dominating the second term in (13) comes from the Coulomb singularity of $W(x)$ which is not fourth power integrable. The singularity can be controlled using the following operator inequality which follows from the diamagnetic inequality $\int|(\boldsymbol{p}+\boldsymbol{A}) \psi|^{2} \mathrm{~d}^{3} x \geq$ $\int|\boldsymbol{p}| \psi||^{2} \mathrm{~d}^{3} x$ and Lemma 2a on p. 708 of [13].

$$
(\boldsymbol{p}+\boldsymbol{A})^{2}-Z /|x| \geq-\left\{\begin{array}{ll}
Z^{2} / 4+(3 / 2) Z R^{-1}, & \text { if }|x| \leq R \\
Z|x|^{-1}, & \text { if }|x| \geq R
\end{array} .\right.
$$

Choose $R=\lambda D_{j}$ and write $(\boldsymbol{p}+\boldsymbol{A})^{2}=\beta(\boldsymbol{p}+\boldsymbol{A})^{2}+(1-\beta)(\boldsymbol{p}+\boldsymbol{A})^{2}$ for some $0<\beta<1$. Then, by scaling,

$$
(\mu / e) \mathcal{T}-W_{\nu} \geq(\mu / e)(1-\beta)(\boldsymbol{p}+\boldsymbol{A})^{2}-(\mu / e) B-\widetilde{W}
$$


where $\widetilde{W}(x, e)=\widetilde{G}_{j}(x, e)+F_{j}(x)$ for $x \in \Gamma_{j}$ with $\widetilde{G}_{j}(x, e)$ defined by

$$
\begin{array}{rll}
\left(Z^{2} e / 4 \beta \mu\right)+3 Z /\left(2 \lambda D_{j}\right) & \text { for } & \left|x-R_{j}\right| \leq \lambda D_{j} \\
Z\left|x-R_{j}\right|^{-1} & \text { for } & \left|x-R_{j}\right|>\lambda D_{j} .
\end{array}
$$

Note that $\widetilde{W}$ depends on $e$.

Again, as in part B, we can use the CLR bound on the second term in (13) to obtain [when $\left.1-\gamma \geq Z^{2} /(4 \beta \mu)\right]$

$$
\begin{aligned}
\sqrt{2} \quad & L_{3}(1-\beta)^{-3 / 2} \int\left\{\int_{\mu}^{\infty}\left[B(x)-\gamma e^{2} / \mu\right]_{+}^{3 / 2} \mathrm{~d} e\right. \\
& \left.+\mu^{-3 / 2} \int_{0}^{\infty}\left[e \widetilde{W}(x, e)-(1-\gamma) e^{2}\right]_{+}^{3 / 2} \mathrm{~d} e\right\} \mathrm{d}^{3} x .
\end{aligned}
$$

First we compute the last integral in (15), which is

$$
\sum_{j=1}^{K} \int_{\Gamma_{j}} \int_{0}^{\infty}\left[e \widetilde{G}_{j}(x, e)+e F_{j}(x)-(1-\gamma) e^{2}\right]_{+}^{3 / 2} \operatorname{ded}^{3} x .
$$

Now split the $\Gamma_{j}$ integral into an inner integral $\left|x-R_{j}\right| \leq \lambda D_{j}$ and an outer integral $\left|x-R_{j}\right|>$ $\lambda D_{j}$. The inner integral yields, using the definitions of $\widetilde{G}_{j}$ and $F_{j}$,

$$
\begin{aligned}
& \frac{3 \pi^{2}}{32}\left(1-\gamma-\frac{Z^{2}}{4 \beta \mu}\right)^{-5 / 2} \int_{0}^{\lambda}\left[\frac{1}{2\left(1-r^{2}\right)}\right. \\
& \left.+\frac{3 Z}{2 \lambda}\right]^{4} r^{2} \mathrm{~d} r D_{j}^{-1} .
\end{aligned}
$$

To bound the outer integral from above we replace $\Gamma_{j}$ by $\boldsymbol{R}^{3}$ and get

$$
\left(3 \pi^{2} / 32\right)(1-\gamma)^{-5 / 2}(\sqrt{Z}+\sqrt{1 / 2})^{8}\left(\lambda D_{j}\right)^{-1} .
$$

Combining (14)-(17) we find that the sum of the negative eigenvalues of $\mathcal{T}-W_{\nu}$ is bounded below by

$$
-a \int W_{\nu}(x)^{5 / 2} \mathrm{~d}^{3} x-b \int B(x)^{2} \mathrm{~d}^{3} x-c \sum_{j=1}^{K} D_{j}^{-1} .
$$

Here $a=q(2 \sqrt{2} / 5) L_{3}(1-\gamma)^{-1}$,

$$
b=q \frac{3 \pi \sqrt{2}}{16} L_{3}(1-\beta)^{-3 / 2}(\mu / \gamma)^{1 / 2}
$$




$$
\begin{aligned}
c= & q \frac{3 \pi^{2} \sqrt{2}}{32} L_{3}(1-\beta)^{-3 / 2} \mu^{-3 / 2}\left\{\frac{(\sqrt{Z}+\sqrt{q / 4})^{8}}{\lambda(1-\gamma)^{5 / 2}}\right. \\
& \left.+\left(1-\gamma-\frac{Z^{2}}{4 \beta \mu}\right)^{-5 / 2} \int_{0}^{\lambda}\left[\frac{q}{4\left(1-r^{2}\right)}+\frac{3 Z}{2 \lambda}\right]^{4} r^{2} \mathrm{~d} r\right\} .
\end{aligned}
$$

To simplify the stability condition we have artificially increased the bounds by recalling that $q \geq 2$ and twice replacing $1 / 2$ by $q / 4$ in the definition of $c$. We choose $\beta=1 / 8, \gamma=1 / 2$, $\lambda=8 / 9$ and $\mu$ so that $b=\left(8 \pi \alpha^{2}\right)^{-1}$. The stability condition $c \leq Z^{2} / 8$ [see (12)] now depends only on the 2 parameters $X=q Z \alpha^{2}$ and $Y=q \alpha$. A straightforward, but lengthy calculation shows that the stability condition holds if $X=X_{0} \equiv 0.082$ and $Y=Y_{0} \equiv 0.12$. The condition is monotone in $Y$, so it holds for $X=X_{0}, Y \leq Y_{0}$. Although our condition does not hold for all $X \leq X_{0}, Y \leq Y_{0}$ we can use the $Z$-monotonicity of $E$ to conclude stability in this range; this proves (4). With the same values of $\beta, \gamma$ and $\lambda$ and with $q=2$ the values $Z=1050$, $\alpha=1 / 137$ also give stability.

To derive (3), note that $W(x) \leq Q\left|x-R_{j}\right|^{-1}$ for $x \in \Gamma_{j}$. Using this bound and replacing $\Gamma_{j}$ by $\boldsymbol{R}^{3}$, one easily obtains $-\sqrt{2} \pi^{2} L_{3} q K Q^{3} \nu^{-1 / 2}-N \nu$ as a lower bound on the $-a \int W_{\nu}^{5 / 2}$ term in (18). Optimizing over $\nu$ yields (3) when $X=X_{0}, Y \leq Y_{0}$. In this case, $Z \geq Z_{0} \equiv 5.7 q$. If $X \leq X_{0}, Y \leq Y_{0}$ and $Z \geq Z_{0}$ we get a lower bound on $E$ by increasing $\alpha$ until $X=X_{0}$, $Y \leq Y_{0}$; this yields (3) with $Q=Q(Z)$. Otherwise, with $Z<Z_{0}$, we use the $Z$-monotonicity of $E$ to conclude (3) with $Q=Q(5.7 q)$.

This work was partially supported by NSF grants PHY90-19433-A04 (E.H.L.), DMS9207703 (M.L.) and DMS92-03829 (J.P.S.).

\section{References}

[1] J. Fröhlich, E. Lieb and M. Loss, Commun. Math. Phys. 104, 251 (1986).

[2] E. Lieb, Bull. Amer. Math. Soc. 22, 1 (1990).

[3] E. Lieb and H.-T. Yau, Commun. Math. Phys. 118, 177 (1988); Phys. Rev. Lett. 61, 1695 (1988).

[4] M. Loss and H.-T. Yau, Commun. Math. Phys. 104, 283 (1986).

[5] J. Avron, I. Herbst and B. Simon, Duke Math. J. 45, 847 (1978); Commun. Math. Phys. 79, 529 (1981). 
[6] I. Daubechies and E. Lieb, Commun. Math. Phys. 90, 497 (1983).

[7] E. Lieb and M. Loss, Commun. Math. Phys. 104, 271 (1986).

[8] J. Conlon, Commun. Math. Phys. 94, 439 (1984).

[9] C. Fefferman and R. de la Llave, Rev. Math. Iberoamericana 2,119 (1986).

[10] J. Combes, R. Schrader and R. Seiler, Ann. Phys. (NY) 111, 1 (1978).

[11] E. Lieb, Proc. Amer. Math. Soc. Symposia in Pure Math. 36, 241 (1980).

[12] E. Lieb and W. Thirring in Studies in Mathematical Physics, edited by E. H. Lieb, B. Simon, A. Wightman (Princeton Univ. Press, Princeton 1976), p. 269.

[13] A. Lenard and F. Dyson, J. Math. Phys. 9, 698 (1968). 